\title{
Perception, experience and knowledge of early physiotherapy in intensive care units of Rome: a survey
}

\author{
Simone Salvitti ${ }^{1}$, Elena Repossini ${ }^{2}$ \\ ${ }^{1}$ Respiratory Rehabilitation Unit, Istituti Clinici Scientifici Maugeri, Pavia; ${ }^{2}$ Pulmonary Outpatient Clinic, Fondazione \\ "Don Gnocchi", Istituto Palazzolo, Milan, Italy
}

\begin{abstract}
Early physiotherapy (EP) programs on critically ill patients in the intensive care unit (ICU) have proven to be safe, feasible and effective. However, despite being considered by all the profes-
\end{abstract}

Correspondence: Simone Salvitti, Respiratory Rehabilitation Unit, Istituti Clinici Scientifici Maugeri, via Salvatore Maugeri 10, 27100 Pavia, Italy.

Tel. +39.0382.592809.

E-mail: simonesalvitti@gmail.com

Key words: early physiotherapy; intensive care unit; critically ill patients; rehabilitation; survey

Contributions: SS, conceived the study and research design, was involved in collection, analysis and interpretation of data, drafted the paper; ER contributed to revising the paper. Both authors read and approved the final manuscript.

Conflict of interest: the authors declare no conflict of interest.

Ethics approval and consent to participate: The approval of the Ethics Committee was not necessary. Participation was voluntary and consent to participate was implicitly expressed by filling in the questionnaires.

Consent for publication: The authors consent to the publication of the manuscript

Availability of data and material: The data used to support the findings of this study are available from the corresponding author upon reasonable request.

Funding: The authors declare that they have not received any kind of funding.

Acknowledgments: Special thanks to Isabel Colado Gimeno and Marta Simonelli for the English translation.

Received for publication: 26 May 2020.

Accepted for publication: 18 September 2020.

${ }^{\circ}$ Copyright: the Author(s), 2020

Licensee PAGEPress, Italy

Monaldi Archives for Chest Disease 2020; 90:1412

doi: 10.4081 monaldi.2020.1412

This article is distributed under the terms of the Creative Commons Attribution Noncommercial License (by-nc 4.0) which permits any noncommercial use, distribution, and reproduction in any medium, provided the original author(s) and source are credited. sionals involved as a fundamental aspect of integrated care, in most cases they are inadequately applied or not performed at all. The main objective of this study is to evaluate the knowledge, perceptions and activities related to EP among physician, nurses and physiotherapists who practice in ICUs of Rome. Obstacles to its application and possible strategies to overcome them are also examined. The study was conducted according to the CHERRIES recommendations. Two questionnaires with 29 questions were created on the Google Form platform and were sent by mail to all the ICUs of public hospitals or accredited private hospitals with the National Health Service in Rome. Thirty (60\%) of the contacted ICUs responded. The physiotherapist is present in $76.7 \%$ of ICUs and carries out its activities mainly during daytime hours $(60.9 \%)$, for less than 3 hours in $39.1 \%$ of cases and only after a request for specialist advice (65.2\%). Only in $4.3 \%$ of cases the service is guaranteed even at weekends but only after a request. All 29 professionals interviewed consider both the presence of a physiotherapist within the interdisciplinary team of the ICU and the implementation of an EP program to be necessary, supporting the positive effects of this program. The most frequently obstacles to the implementation of an EP program are clinical instability (69\%), low priority to EP practice $(62.1 \%)$, cardiovascular instability $(58.6 \%)$ and lack of experience $(58.6 \%)$. The most frequently indicated strategies to overcome these obstacles are to organize interdisciplinary briefings $(86.2 \%)$, to avoid excluding patients from treatment without specific motivation $(75.9 \%)$, to invest in staff training (75.9\%), to use protocols and guidelines (58.6\%). EP is not currently exploited to its full potential due to lack of funds, culture, experience, training and a hospital organization that limits the provision of effective patient care and efficient service to the National Health Service.

\section{Introduction}

Traditionally the measure of the quality of treatment in Intensive Care Unit (ICU) was focused on mortality. Over time, the importance of the ICU has no longer been limited only to the survival of the patient, but also to the limitation of the onset of complications [1], the preservation of functional capabilities and the maintenance of quality of life [2]. This happens because both hospital and local health care systems are constantly under pressure because they are called on to provide high quality services while reducing costs [3].

The term early physiotherapy (EP) means the beginning of physiotherapy treatment within $48 \mathrm{~h}$ from ICU admission. Many studies showed that EP interventions are feasible, safe and effective $[4,5]$ and can effectively improve the patient's mental health 
status, prevent the formation of bedsores and the related infections, enhance physical abilities, prevent ICU-Acquired Weakness, reduce the time of mechanical ventilation (MV) and associated complications, facilitate the weaning process from MV also due to the possibility of reducing sedation, decreasing hospital length of stay, improving outcomes and the quality of life at discharge and reduce the mortality rate [2-14]. A general improvement in the patient's health at discharge, together with the reduction of hospital length of stay, leads to an improvement in the quality of ICU $[10,15]$ and to a cost reduction of both the department $[3,4,8,12$, 14-16] and territorial health services. All these evidences confirm the fundamental role of the physiotherapist in ICU as an expert in the evaluation of functions and treatment of the respiratory and musculoskeletal systems in a view of progression, effectiveness and efficiency of therapeutic exercise.

Unfortunately, however, critical patients are often considered too sick or unfit to participate to EP interventions; not only in Italy $[10,17]$, it is rare to find a dedicated physiotherapist who is a permanent member of the interdisciplinary team of an ICU, despite the EP is considered a fundamental aspect by all professionals involved. The cause of this contradiction is to be found not only in the lack of training and experience of physiotherapists, who occasionally work in ICU and rarely perform activities beyond passive and active mobilizations and bedside positioning of the patient, especially in MV [5], but also in the culture and organization of both the department and the healthcare facility. Culture includes the attitudes, opinions and prejudices that all staff have in common and determine how the team embraces change and maintains it over time [18]. To understand the obstacles and to find possible strategies to overcome them is useful to introduce EP as a routine clinical practice in ICU [19] and to change the mindset of a department with activities based on the achievement of shared goals. This cultural transformation, combined with patient-centred care, leads to the improvement of patient health, increased staff satisfaction and an increase in the quality of the department and the service provided [11].

Many studies highlighted the benefits, safety and possibilities to put the EP program into practice [2,20], but few investigated the perception, knowledge and experience of staff regarding this practice.

The primary aim of this study is to evaluate knowledge, perceptions and activities regarding EP among physician, nurses and physiotherapists practicing in ICUs of Rome. Obstacles to its application and possible strategies to overcome them are also examined.

\section{Materials and Methods}

\section{Survey development}

This survey was conducted from June 2017 to February 2018 (9 months) and was divided into three different phases: i) literature review; ii) creation of a list of telephone numbers and email addresses of the ICUs coordinators in Rome; iii) creation and administration of two questionnaires.

The literature review was conducted on MEDLINE (through PubMed), Cochrane Library, Google Scholar and PEDro and it was necessary to include in the second questionnaire all the questions related to the effects of $\mathrm{EP}$, the causes that may hinder this activity and the possible strategies to be implemented to overcome them.

Due to the impossibility of finding an existing mailing list containing all the email addresses of the participants, these were acquired through the websites of the hospitals or by telephone con- tact. This study did not require the approval of the Ethics Committee.

Google Forms web application (Google LLC, Mountain View, CA, USA) was used for the creation, submission and data acquisition of the two questionnaires. These were composed by means of what had been collected and analysed in the literature review and were submitted to a pool of experts consisting of physicians, nurses and physiotherapists, who did not consider it necessary to make any changes. The electronic system was successfully tested by 20 physiotherapists before the beginning of the study.

Participants were contacted by email. The introductory email contained information on the purpose of the study, the identity of the study supervisor, the estimated average time needed to complete the questionnaires and on the acquisition and processing of data in a totally anonymous form. The platform for filling in questionnaires could only be accessed via a link sent by email, which made the survey closed. It was possible to fill in the questionnaires from September to December 2017 (3 months) and the data entered in the questionnaires were automatically acquired and recorded by the web application.

The study was not advertised in any way either for free or for a fee, participation in the study was totally voluntary and participants did not receive any kind of incentive to fill in the questionnaires.

The e-mail addresses of the participants were entered and stored automatically by the application in order to send reminder messages every 15 days to those who did not answer. Each participant could only fill in the questionnaires once without the need to register and/or login to the web application platform. The survey provider's account was only accessible by the survey supervisor for ensuring data protection and the anonymity of the participants.

\section{Survey design}

The first questionnaire, consisting of 21 questions, was addressed to the ICUs coordinators in Rome and aimed to collect general information about the hospital, the ICU department and the staff working there. The second questionnaire, consisting of 8 questions and sent only to the ICUs who had already completed the first questionnaire, was addressed to physicians, nurses and physiotherapists working in these units and aimed to highlight the perception, knowledge and state of the art of EP in ICUs as well as the obstacles to its application and possible strategies to overcome them based on what is reported in the literature (Supplementary Material).

The questions were not randomized and some of them were adaptive. The complete survey is composed of 29 questions distributed in 8 sections (minimum 1 and maximum 10 questions per section) excluding the 2 final sections, one per questionnaire, which contained a space for the optional addition of any notes. Only the ICUs coordinators had the possibility to answer all the questions, while the other participants could only fill in the second questionnaire. The questions in the first questionnaire were reduced from 21 to 15 if the participant stated that he did not cooperate with physiotherapists within his department. All questions required a mandatory answer except, of course, those that were not shown, so that there were no incomplete questionnaires. Questions that required an open answer and therefore the input of non-standardized values were equipped with a control system that generated an error message if incorrect and/or null values were input. It was not possible to record the time taken to complete the questionnaires to check which of them had been completed with an above-average time.

Participants were able to review and modify their responses until the closure of the survey and the sending of the questionnaire was confirmed. It was not possible to review or modify the answers after the closure of the questionnaires. 


\section{Data analysis}

In the case of multiple responses, these were identified by the email address of origin and the participant was asked which version he wanted to confirm. This was the only way to check multiple responses. Descriptive statistics are presented as counts and percentages, mean and standard deviation.

For the conduct of this study, the CHERRIES recommendations (Checklist for Reporting Results of Internet E-Surveys) for improving the quality of surveys conducted via web platforms were followed [21].

\section{Results}

In August, 50 ICUs of public or accredited private hospitals of Rome were surveyed and contacted through the unit nursing coordinators. Based on the correctly completed questionnaires sent within the study deadline, 30 out of a total of 50 ICUs were recruited $(60 \%)$ (Figure 1). The description of the enrolled ICUs is in Table 1.

The analysis of the data shows that the physiotherapist is present in 23 of $30(76.7 \%)$ of the ICUs examined but that in $65.2 \%$ of cases he carries out his activities only after a physician's request; however, there are also situations in which the physiotherapist can carry out an EP program because is structurally integrated into the interdisciplinary team of the ICU $(26.1 \%)$ and in which this professional provides his services independently $(8.7 \%)$. It should be noted that most of the time physiotherapists work in ICU departments 6 days a week $(60.9 \%)$ for between 1 and 3 hours $(39.1 \%)$ with no service in the afternoon $(60.9 \%)$ or on Sundays $(100 \%)$ except when required $(4.3 \%)$. In 6 ICUs the physiotherapist is present 5 days a week (26.1\%), in $1 \mathrm{ICU}$ he is present 3 days a week $(4.3 \%)$ and in 2 ICUs he works occasionally, if necessary $(8.7 \%)$. It is also noted that there is 1 unit where the physiotherapist works 6 or more hours a day $(4.3 \%)$, in 2 ICUs he is present for about 3-6 hours (8.7\%), in 3 ICUs he works about 3 hours $(13 \%)$ and 7 ICUs where he stays about 1 hour $(30.4 \%)$. Considering instead the data regarding the presence of this professional in ICU also in the afternoon and at weekends, it is

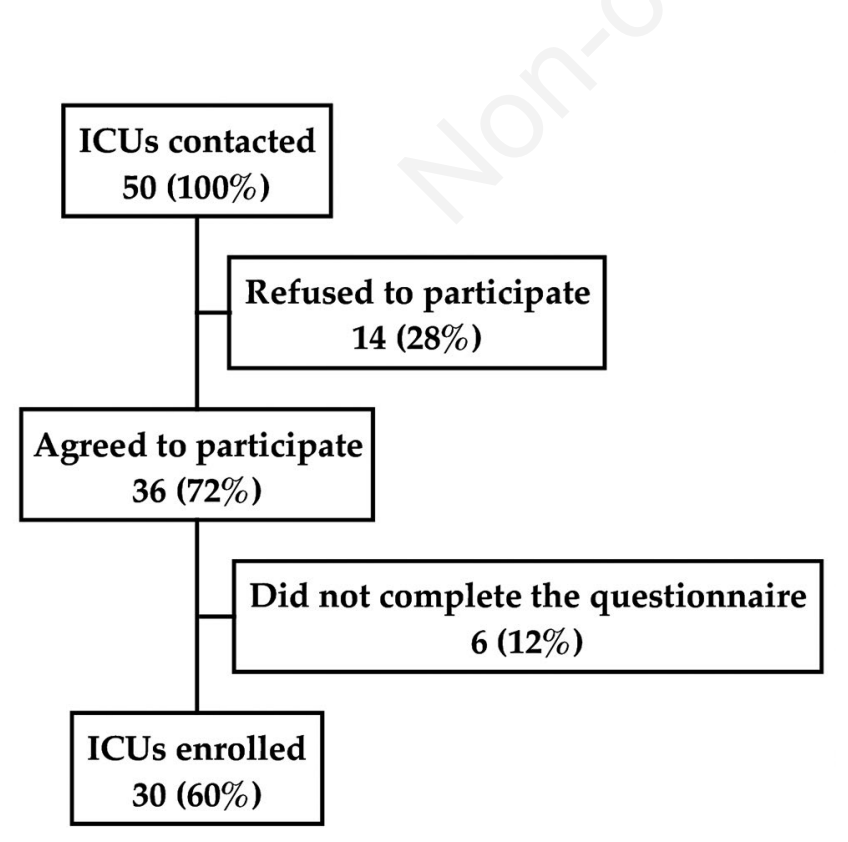

Figure 1. Enrolment process. ICU, Intensive Care Unit. evident that in 6 ICUs the afternoon activity takes place only from Monday to Friday (26.1\%), in 2 ICUs he works in the afternoon also on Saturday $(8.7 \%)$ and in 1 ICU the physiotherapist stays beyond the common working hours only on request (4.3\%). In 6 ICUs the physiotherapist does not guarantee the service on Saturday and Sunday $(26.1 \%)$ and in 1 ICU he works on weekends only on request $(4.3 \%)$.

Regarding ICU's activities, it should be noted that most of them are carried out by physicians and nurses (Figure 2). Physiotherapists carry out activities that are almost exclusively related to the orthopedic field of physiotherapy, such as passive (48.9\%) and active mobilization (53.7\%), get the patient out of bed $(35.9 \%)$ and walking $(50 \%)$. Concerning respiratory therapies, physiotherapists perform assisted cough (36.1\%), airway clearance techniques $(34.5 \%)$ or airway clearance with positive pressures $(30 \%)$, pulmonary function tests $(31 \%)$. Other activities less performed by physiotherapists include: continuous lateral rotation therapy $(16.1 \%)$, airway suctioning $(15.2 \%)$, spontaneous breathing trial during weaning from MV (13.6\%), tracheostomy cannula cleaning $(12.5 \%)$, aerosol therapy $(12.1 \%)$, pulmonary auscultation $(8.1 \%)$, oxygen therapy $(7.4 \%)$, airway humidification $(5.3 \%)$, choose proper interface for NIV (3.4\%), use of Ambu bag (3.1\%), decannulation (2.9\%) and NIV adaptation process $(2.7 \%)$.

The sample respondent to the second questionnaire was composed as follows:

- 8 physicians $(27.6 \%)$ - out of a total of $312(2.6 \%)$

- 11 nurses $(37.9 \%)$ - out of a total of $684(1.6 \%)$

- 10 physiotherapists (34.5\%) - out of a total of $41(24.4 \%)$

All the 29 professionals interviewed, from 17 of the 30 enrolled ICUs (56.7\%), consider both the presence of a physiotherapist within the interdisciplinary team of the ICU department and the implementation of an EP program to be necessary, as they believe it may have positive effects on the clinical course of the critical patient. $55.2 \%$ of them ( 6 physicians, 5 nurses and 5 physiotherapists) state that they are aware of the effects of EP reported in the literature, while the remaining 44.8\% (2 physicians, 6 nurses and 5 physiotherapists) deny knowing them.

With regard to the effects of EP perceived by the various professionals figures (Figure 3 ) it is recorded that the most frequent choices were: enhancement of physical abilities $(79.3 \%)$, prevention of bed sores $(75.9 \%)$, improvement of physical health condition (72.4\%), making it easier for the patient to get out of bed $(65.5 \%)$, prevention of muscle deconditioning $(65.5 \%)$, prevention of physical deconditioning $(65.5 \%)$, prevention of muscle contracture and retraction $(65.5 \%)$, reduction of ICU length of stay (62.1\%), prevention of ICU acquired weakness $(58.6 \%)$, prevention of deep vein thrombosis (55.2\%), facilitation of the weaning from MV (48.3\%), reduction of time on MV (48.3\%), reduction of airway infections (48.3\%), improvement of mental health condition $(48.3 \%)$, improvement of quality of life $(41.4 \%)$, reduction of department costs (41.4\%), improvement of ventilation and gas exchange (37.9\%), improvement of efficiency of ICU (34.5\%), reduction of delirium $(31 \%)$, reduction hospital length of stay $(31 \%)$, prevention of bone demineralization $(24.1 \%)$, reduction of tracheotomy $(24.1 \%)$, reduction of mortality $(20.7 \%)$, reduction territorial health services costs $(20.7 \%)$, reduction of reintubation $(17.2 \%)$, reduction of ICU readmission (13.8\%), reduction of caregiving burden (13.8\%) and improvement of glycemic control (10.3\%).

The perceived obstacles to the EP are shown in Table 2. The strategies proposed to overcome them have been divided into categories, differentiating them into patient related, ward culture related, hospital related and related to management of ICU activities (Table 3). 


\section{Discussion}

Although the general picture described by the data collected is not fully representative of the totally of the sample surveyed due to the few responses received, in according with Bakhru et al. [10] and Grammatopoulou et al. [17], it highlights that in ICU the physiotherapist is a professional figure who in most cases provides his services only after a physician's request, as he is not structurally integrated in the interdisciplinary team of the department. Our data, however, disagree with another study by Bakhru and colleagues [22] who describes a greater presence of dedicated physiotherapists in ICU.

The lack of physiotherapists and the inadequate physiotherapists/beds ratio has also been highlighted by other studies $[23,24]$. Even those who described a ratio similar to ours (about 1 physiotherapist per 8 beds) reported that many patients were not mobilized early due to barriers such as sedation and intubation $[22,25,26]$. The most frequently reported obstacles to EP and strategies to overcome them are in agreement with the literature [10,27-30]. The paucity of physiotherapists combined with lack of experience and expertise does not make EP any easier. Furthermore, unlike other professionals who work in the ICU, these data show that physiotherapists do not ensure continuity of care in the afternoon or at weekends [17]. This type of work shift organization is not compatible with the implementation of an EP program as the patient could work only a few hours a day, usually the more activity intensive ones, spending the remaining hours in bed [31].
In an overview it can be seen that the most frequent answers regarding the effects of an EP program are related to the effectiveness in improving the patient's psychophysical conditions and then progressively less linked to the efficiency of care and the reduction in the use of both economic and non-economic resources. According to the scientific evidence, despite the thought of the professionals examined, an EP program can reduce mortality [7,12], the probability of readmission to ICU [32] and the probability of reintubation [33]. Moreover, it has been seen that through EP it is possible to improve glycemic control [16] and, more generally, functional capacities and quality of life, which suggests that patients, once discharged at home, may need reduced territorial health care, thus reducing both the costs and the work of caregivers.

Our study shows that clinical instability is an obstacle to EP in many cases, despite the literature advises not to exclude patients from treatment a priori but to assess on a case-by-case basis in order to perform the highest possible level of rehabilitation safely for both the patient and the professional. A possible strategy is the use of the ICU Mobility Scale [34] which evaluates the highest possible level of mobilization and perform continuous monitoring of vital parameters during treatment as suggested by Harris and Shahid [35]. It is also possible to safely mobilize the patient in the presence of endotracheal devices [15], femoral catheters [36], CRRT [37] and ECMO [38].

It has emerged that one of the obstacles related to the culture of ICU is deep sedation which is one of the main causes of psychophysical deterioration of patients in ICU [39] besides entailing

Table 1. Description of enrolled ICUs.

\begin{tabular}{|c|c|c|c|}
\hline & Mean (SD) & Professional/bed ratio & n $(\%)$ \\
\hline Beds & $10.93(4.31)$ & - & $328(100)$ \\
\hline Physicians & $10.4(4.12)$ & 0.95 & $312(28.8)$ \\
\hline Residents & $1.57(1.96)$ & 0.14 & $47(4.3)$ \\
\hline Nurses & $22.8(6.73)$ & 2.09 & $684(63.1)$ \\
\hline Physiotherapists & $1.37(1.27)$ & 0.13 & $41(3.8)$ \\
\hline $\begin{array}{l}\text { Hospitals enrolled } \\
\text { Public } \\
\text { Private accredited with the National Health Service }\end{array}$ & & & $\begin{array}{l}30(100) \\
22(73.3) \\
8(26.7)\end{array}$ \\
\hline $\begin{array}{l}\text { Hospital beds } \\
<250 \\
250-400 \\
401-700 \\
>700\end{array}$ & & & $\begin{array}{c}3(10) \\
3(10) \\
9(30) \\
15(50)\end{array}$ \\
\hline $\begin{array}{l}\text { Specialty of ICU } \\
\text { General } \\
\text { Cardiac surgery } \\
\text { Neurosurgical } \\
\text { Postoperative } \\
\text { Thoracic } \\
\text { Organ transplants } \\
\text { Stroke Unit } \\
\text { Respiratory } \\
\text { Cardiac }\end{array}$ & & & $\begin{array}{l}9(30) \\
3(10) \\
3(10) \\
1(3.3) \\
1(3.3) \\
2(6.8) \\
1(3.3) \\
1(3.3) \\
9(30)\end{array}$ \\
\hline $\begin{array}{l}\text { Level of care } \\
\text { First } \\
\text { Second }\end{array}$ & & & $\begin{array}{c}21(70) \\
9(30)\end{array}$ \\
\hline Management of IMV & & & $27(90)$ \\
\hline Management of NIV & & & $29(96.7)$ \\
\hline
\end{tabular}

ICU, Intensive Care Unit; IMV, invasive mechanical ventilation; NIV, non-invasive ventilation. 
considerable costs [1] and delaying physiotherapy [40]. Among the first studies that found a possible link between deep sedation and the adverse effects of immobility is that of De Jonghe et al. [41] who, with the ATICE algorithm, were able to reduce the time in $\mathrm{MV}$, ICU length of stay and the appearance and duration of delirium. Kress and colleagues [42] showed that signs of post-traumatic stress disorder were less frequent in patients who stopped the infusion of sedatives daily than in patients receiving the usual therapy.

Respondents said that another patient related obstacle to EP is the presence of delirium although it is reported in the literature that one of the non-pharmacological delirium treatment strategies for ICU patients include frequent space-time reorientation, sleep

\section{Physicians $\square$ Nurses Physiotherapists}

Passive mobilization

Active mobilization

Assisting the patient to walk

Getting patients out of bed

Assisted cough

Airway clearance techniques

Airway clearance with PEP

Continuous lateral rotation therapy

Airway suctioning

Aerosol therapy

Pulmonary function tests

Tracheostomy cannula cleaning

SBT during weaning from MV

Pulmonary auscultation

Oxygen therapy

Decisions on airway humidification

Use of Ambu bag

Choose proper interface for NIV

NIV adaptation process

Decannulation

Ventilator circuit replacement

Check of ventilator circuit

ABG

Active respiratory gas humidification during MV

Monitoring of ventilator settings during MV

Check of ventilator settings before MV

Estubation

Weaning decision making

Ventilator settings

Pain management

Sedation management
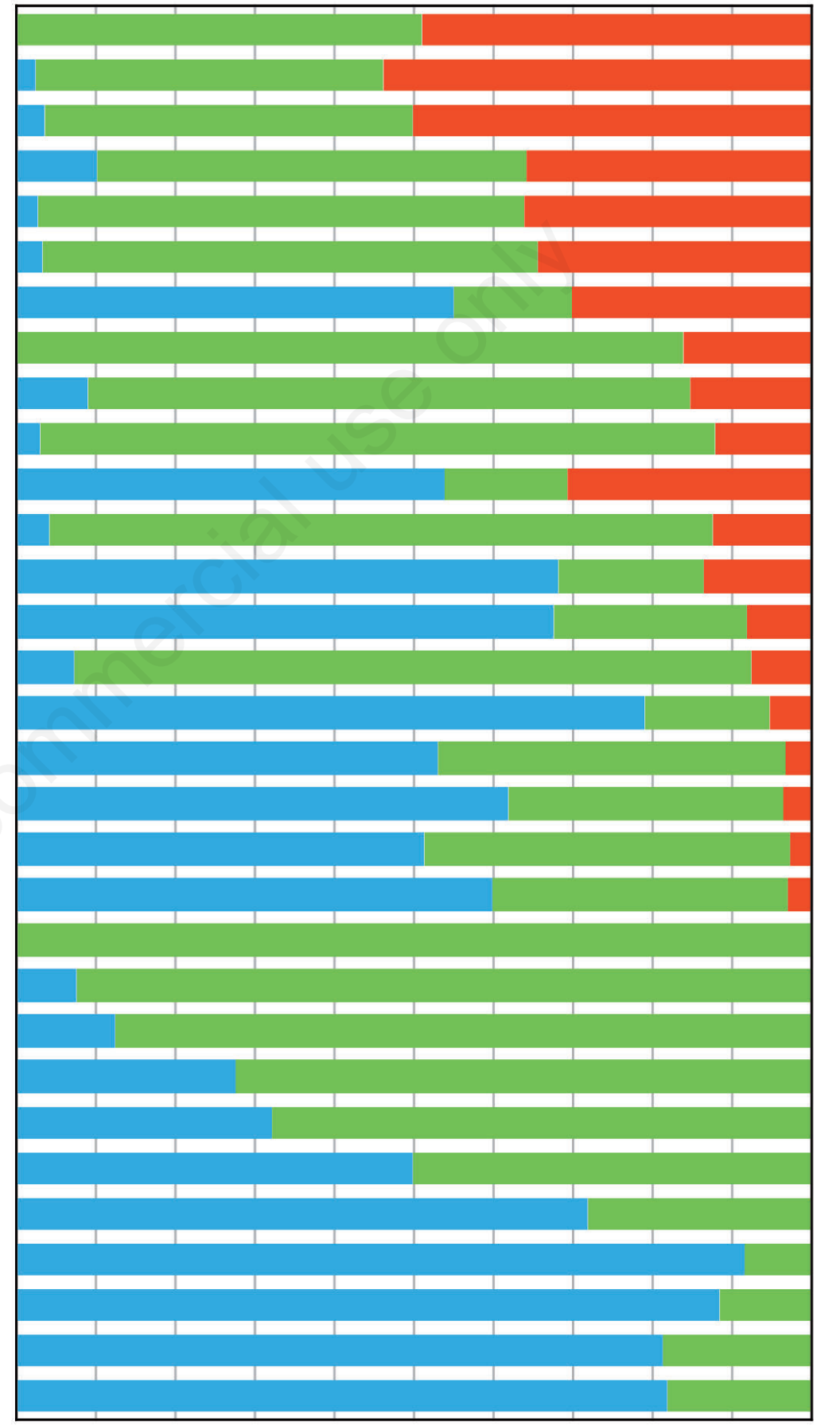

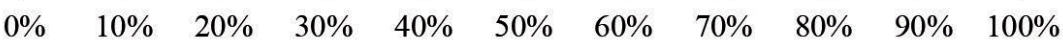

Figure 2. Activities carried out by ICUs professionals. PEP, positive expiratory pressure; SBT, spontaneous breathing trial; MV, mechanical ventilation; NIV, non-invasive ventilation; ABG: arterial blood gas. 
restoration, EP and modification and integration of environmental stimuli [43]. Minimization and daily interruption of sedation can be combined with EP [5] as demonstrated by the ABCDE bundle which led to increased out-of-bed activity, improving patient outcome and reducing delirium episodes [44]. To implement an EP program in an ICU, it is necessary to create a strong and fruitful collaboration between the team members of the department, as Bronstein suggests [45]. In accordance with what expressed by the professionals interviewed, to facilitate and speed up the approach of patients with rehabilitation it is necessary to draw up protocols and guidelines that can direct and guide all operators towards the delivery of the most effective and efficient therapies available [46]. Given the complexity of the management of the change of approach to the patient in order to create a culture that favors the EP, in accordance with the answers of the respondents, it is necessary to design projects that involve all the operators of the hospital such as managers, administrators, coordinators, physicians, paramedics, auxiliaries, etc. Over the years, many ICU quality improvement projects have been proposed both by doctors and by other healthcare professionals, with truly remarkable results $[9,11,14,46-49]$.

It is natural to assume that every project has implementation costs and, as in our case, these costs are often perceived as an obstacle to the realization of the project. The costs of an EP program are divided into three main categories: personnel, training and equipment [4]. Most of the costs would concern the recruitment and training of staff, but these should be incurred only once [50] and, would be more than justified by the better results on patients' outcomes at discharge, the reduction of ward costs $[8,16]$, the improvement of the quality of service offered and the progressive increase in the number of accesses to ICU. The healthcare sec-

Table 2. Perceived obstacles to early physiotherapy (full data in Supplementary Material).

\begin{tabular}{|c|c|c|c|c|}
\hline Obstacles to early physiotherapy & Physicians n (\%) & Nurses n (\%) & Physiotherapists n (\%) & Total n (\%) \\
\hline \multicolumn{5}{|l|}{ Patient } \\
\hline Clinical instability & $5(62.5)$ & $10(90.9)$ & $5(50)$ & $20(69)$ \\
\hline Cardiovascular instability & $3(37.5)$ & $8(72.7)$ & $6(60)$ & $17(58.6)$ \\
\hline Delirium & $4(50)$ & $6(54.5)$ & $6(60)$ & $16(55.2)$ \\
\hline Intubation & $4(50)$ & $7(63.6)$ & $5(50)$ & $16(55.2)$ \\
\hline Orthopedic diseases or limitations & $5(62.5)$ & $5(45.5)$ & $5(50)$ & $15(51.7)$ \\
\hline Respiratory instability & $3(37.5)$ & $7(63.6)$ & $5(50)$ & $15(51.7)$ \\
\hline Psychomotor agitation & $4(50)$ & $5(45.5)$ & $5(50)$ & $14(48.3)$ \\
\hline Inadequate analgesia & $4(50)$ & $3(27.3)$ & $6(60)$ & $13(44.8)$ \\
\hline Risk of dislocation of catheters, tubes and cables & $3(37.5)$ & $5(45.5)$ & $5(50)$ & $13(44.8)$ \\
\hline Patient refusal & $4(50)$ & $5(45.5)$ & $4(40)$ & $13(44.8)$ \\
\hline \multicolumn{5}{|l|}{ Culture } \\
\hline Low priority to early physiotherapy & $5(62.5)$ & $5(45.5)$ & $8(80)$ & $18(62.1)$ \\
\hline Lack of experience & $6(75)$ & $4(36.4)$ & $7(70)$ & $17(58.6)$ \\
\hline Continuous deep sedation & $6(75)$ & $5(45.5)$ & $5(50)$ & $16(55.2)$ \\
\hline Insufficient knowledge of scientific evidence & $6(75)$ & $2(18.2)$ & $6(60)$ & $14(48.3)$ \\
\hline Inadequate staff training & $6(75)$ & $2(18.2)$ & $6(60)$ & $14(48.3)$ \\
\hline Lack of knowledge of the benefits of treatment by the staff & $5(62.5)$ & $3(27.3)$ & $6(60)$ & $14(48.3)$ \\
\hline Lack of medical recommendations & $2(25)$ & $3(27.3)$ & $7(70)$ & $12(41.4)$ \\
\hline Lack of medical authorization & $3(37.5)$ & $2(18.2)$ & $7(70)$ & $12(41.4)$ \\
\hline Discordant views about the benefits of EP & $2(25)$ & $2(18.2)$ & $4(40)$ & $8(27.6)$ \\
\hline Increase of costs & $4(50)$ & $1(9.1)$ & $2(20)$ & $7(24.1)$ \\
\hline \multicolumn{5}{|l|}{ Hospital } \\
\hline Absence of physiotherapists & $6(75)$ & $6(54.5)$ & $5(50)$ & $17(58.6)$ \\
\hline Lack of founds & $3(37.5)$ & $5(45.5)$ & $6(60)$ & $14(48.3)$ \\
\hline Limited staff & $3(37.5)$ & $4(36.4)$ & $4(40)$ & $11(37.9)$ \\
\hline Absence of computerization of medical records & $4(50)$ & $3(27.3)$ & $4(40)$ & $11(37.9)$ \\
\hline Lack of material and equipment & $2(25)$ & $2(18.2)$ & $3(30)$ & $7(24.1)$ \\
\hline \multicolumn{5}{|l|}{ Management } \\
\hline Absence of protocol & $6(75)$ & $3(27.3)$ & $8(80)$ & $17(58.6)$ \\
\hline Absence of guideline & $6(75)$ & $2(18.2)$ & $7(70)$ & $15(51.7)$ \\
\hline Lack of well-defined roles and responsibilities & $4(50)$ & $4(36.4)$ & $5(50)$ & $13(44.8)$ \\
\hline Poor communication between staff & $3(37.5)$ & $5(45.5)$ & $4(40)$ & $12(41.4)$ \\
\hline Lack of work coordination & $3(37.5)$ & $3(27.3)$ & $5(50)$ & $11(37.9)$ \\
\hline
\end{tabular}


tor is not immune to periods of economic crisis and therefore it is necessary not only to disinvest on treatments that are not scientifically justified and limited in effectiveness and efficiency, but also to reallocate resources and redistribute them into healthcare services that can improve the health status of patients, possibly further reducing costs.

The strength of this study is to have highlighted the criticality related to the low presence of physiotherapists in the ICUs of Rome who work in these departments mainly after a physician's request and not guaranteeing the service in the afternoon and weekend. It also highlights how some activities such as mobilization, airway clearance, pulmonary function tests, management of mechanical ventilation and other are performed by physiotherapists only in some cases. It also emerged that the professionals interviewed recognize the lack of staff, material, knowledge, experience, protocols and guidelines as elements that hinder an EP program and they propose to overcome them through training, investment, involvement of all professionals working in the hospital and creation of protocols and guidelines that define responsibilities and actions of each professional.

The limits of the study are the small sample size and the geographical connotation which limits the generalizability of the results to other regions. In the future we could repeat a survey like this one, perhaps adopting strategies to increase the response rate such as sending reminders, using hand-printed envelopes and offering small incentives for those who want to participate in the study.

EP is not currently exploited to its full potential due to lack of funds, culture, experience, training and a hospital organization that limits the provision of effective patient care and efficient service to the National Health Service. In order to recognize physiotherapy as a fundamental part of the essential care of the critical patient, it is essential to undertake a long path in every ICU, from the awareness of the effectiveness of an intervention to its implementation, changing the mindset and consequently the modus operandi of the ICU team and the organizational, administrative and managerial hospital staff.

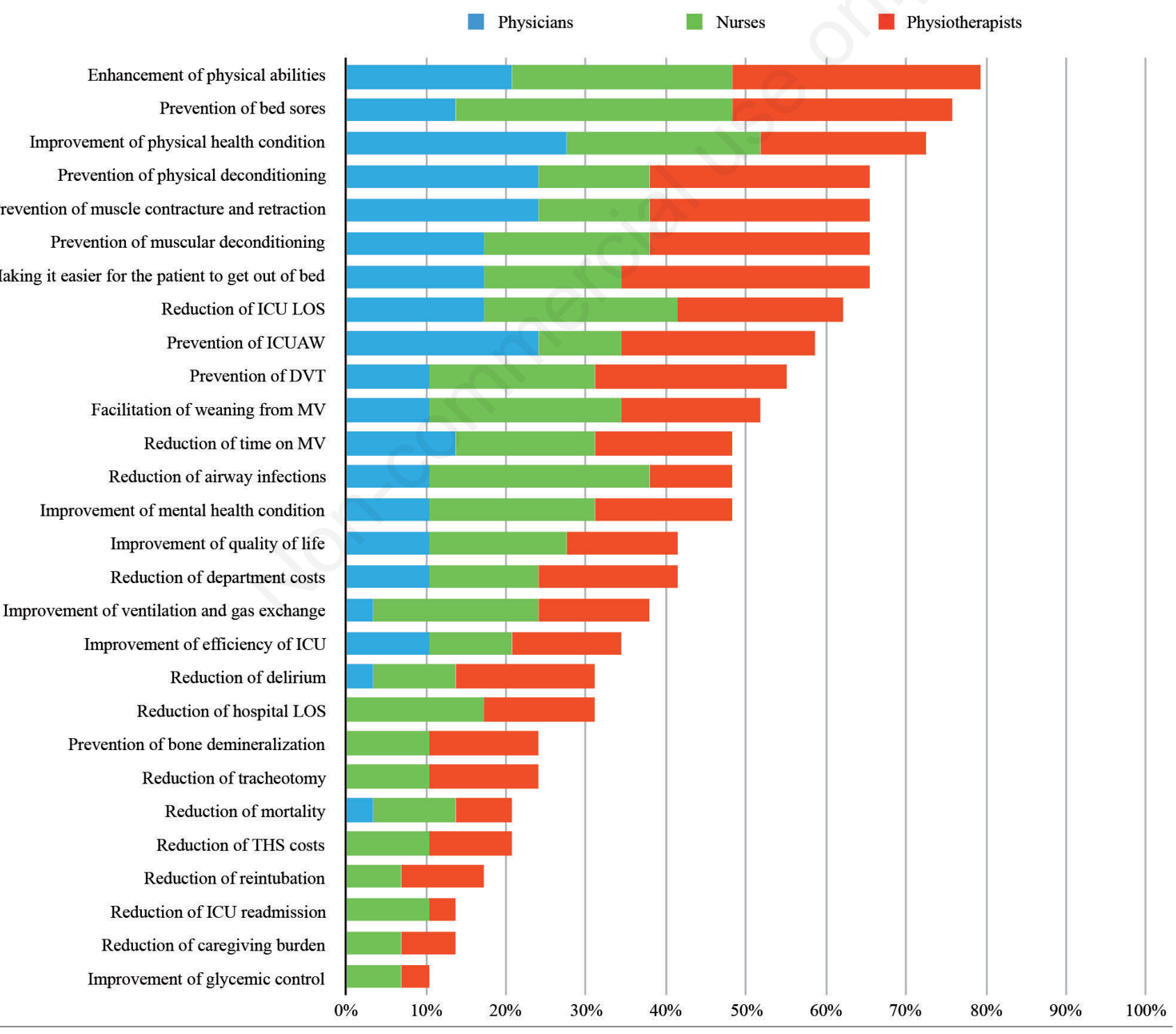

Figure 3. Perception and knowledge of effects of early physiotherapy. ICU, intensive care unit; LOS, length of stay; ICUAW, intensive care unit acquired weakness; DVT, deep vein thrombosis; MV, mechanical ventilation; THS, territorial health services. 
Table 3. Strategy to overcome the obstacles to early physiotherapy (full data in Supplementary Material).

\begin{tabular}{|c|c|c|c|c|}
\hline Facilitations to early physiotherapy & Physicians n (\%) & Nurses n (\%) & Physiotherapists n (\%) & Total n (\%) \\
\hline \multicolumn{5}{|l|}{ Patients } \\
\hline Interdisciplinary briefing & $6(75)$ & $10(90.9)$ & $9(90)$ & $25(86.2)$ \\
\hline Avoid excluding a priori but assess patents case-by-case & $6(75)$ & $9(81.8)$ & $7(70)$ & $22(75.9)$ \\
\hline Listen and encourage the patient & $3(37.5)$ & $7(63.6)$ & $7(70)$ & $17(58.6)$ \\
\hline Use protocols and/or guidelines & $5(62.5)$ & $6(54.5)$ & $6(60)$ & $17(58.6)$ \\
\hline Establish safety ranges for the patient's vital signs & $5(62.5)$ & $6(54.5)$ & $6(60)$ & $17(58.6)$ \\
\hline Ensure analgesic coverage before treatment & $4(50)$ & $6(54.5)$ & $6(60)$ & $16(55.2)$ \\
\hline Daily screening of patients by physiotherapists & $3(37.5)$ & $6(54.5)$ & $6(60)$ & $15(51.7)$ \\
\hline Gradual and progressive activities & $4(50)$ & $5(45.5)$ & $5(50)$ & $14(48.3)$ \\
\hline Daily evaluation of delirium & $3(37.5)$ & $5(45.5)$ & $6(60)$ & $14(48.3)$ \\
\hline Daily assessment and start treatment soon as possible & $3(37.5)$ & $6(54.5)$ & $4(40)$ & $13(44.8)$ \\
\hline \multicolumn{5}{|l|}{ Culture } \\
\hline Invest in staff training & $6(75)$ & $7(63.6)$ & $9(90)$ & $22(75.9)$ \\
\hline Learn about of benefits of early physiotherapy & $4(50)$ & $7(63.6)$ & $5(50)$ & $16(55.2)$ \\
\hline Create protocol for sedation & $5(62.5)$ & $5(45.5)$ & $6(60)$ & $16(55.2)$ \\
\hline Organize refresher courses & $4(50)$ & $5(45.5)$ & $7(70)$ & $16(55.2)$ \\
\hline Cross-training of staff with interdisciplinary lessons & $3(37.5)$ & $5(45.5)$ & $7(70)$ & $15(51.7)$ \\
\hline Recruit physiotherapists to create stable leadership & $4(50)$ & $5(45.5)$ & $5(50)$ & $14(48.3)$ \\
\hline Set as target a mild sedation & $4(50)$ & $4(36.4)$ & $5(50)$ & $13(44.8)$ \\
\hline Creating an EP program to achieve shared goals & $3(37.5)$ & $5(45.5)$ & $5(50)$ & $13(44.8)$ \\
\hline Learn how to quickly identify suitable patients for treatment & $4(50)$ & $3(27.3)$ & $6(60)$ & $13(44.8)$ \\
\hline Perform daily sedation assessments & $3(37.5)$ & $4(36.4)$ & $5(50)$ & $12(41.4)$ \\
\hline \multicolumn{5}{|l|}{ Hospital } \\
\hline Involve organizational, administrative and managerial staff & $3(37.5)$ & $6(54.5)$ & $6(60)$ & $15(51.7)$ \\
\hline Train staff in the appropriate use of the available material & $5(62.5)$ & $4(36.4)$ & $5(50)$ & $14(48.3)$ \\
\hline Recruit more physiotherapists and other rehabilitation specialists & $2(25)$ & $5(45.5)$ & $4(40)$ & $11(37.9)$ \\
\hline Create functional departments for interdisciplinary management of patient & $3(37.5)$ & $4(36.4)$ & $4(40)$ & $11(37.9)$ \\
\hline \multicolumn{5}{|l|}{ Management } \\
\hline Stimulate communication, confrontation and interdisciplinary cooperation & $3(37.5)$ & $4(36.4)$ & $5(50)$ & $12(41.4)$ \\
\hline Creation of a mobility team & $3(37.5)$ & $4(36.4)$ & $4(40)$ & $11(37.9)$ \\
\hline Improve work coordination & $2(25)$ & $4(36.4)$ & $5(50)$ & $11(37.9)$ \\
\hline Create protocols and guidelines to ensure a quick and safe start of treatme & $1(12.5)$ & $4(36.4)$ & $5(50)$ & $10(34.5)$ \\
\hline
\end{tabular}

EP, early physiotherapy.

\section{References}

1. Kress JP. Sedation and Mobility: Changing the Paradigm. Crit Care Clin 2013;29:67-75.

2. Kayambu G, Boots R, Paratz J. Physical therapy for the critically ill in the ICU: a systematic review and meta-analysis. Crit Care Med 2013;41:1543-54.

3. Hunter A, Johnson L, Coustasse A. Reduction of intensive care unit length of stay: the case of early mobilization. Health Care Manag 2014;33:128-35.

4. Cameron S, Ball I, Cepinskas G, et al. Early mobilization in the critical care unit: A review of adult and pediatric literature. J Crit Care 2015;30:664-72.

5. Schweickert WD, Pohlman MC, Pohlman AS, et al. Early physical and occupational therapy in mechanically ventilated, critically ill patients: a randomised controlled trial. Lancet 2009;373:1874-82.
6. Lai CC, Chou W, Chan KS, et al. Early mobilization reduces duration of mechanical ventilation and intensive care unit stay in patients with acute respiratory failure. Arch Phys Med Rehab 2017;98:931-9.

7. Sawada Y, Sasabuchi Y, Nakahara Y, et al. Early rehabilitation and in-hospital mortality in intensive care patients with community-acquired pneumonia. Am J Crit Care 2018;27:97-103.

8. Hsieh SJ, Otusanya O, Gershengorn HB, et al. Staged implementation of $\mathrm{ABCDE}$ bundle improves patient outcomes and reduces hospital costs. Crit Care Med 2019;47:885.

9. McWilliams D, Weblin J, Atkins G, et al. Enhancing rehabilitation of mechanically ventilated patients in the intensive care unit: a quality improvement project. J Crit Care 2015;30:13-18.

10. Bakhru RN, Wiebe DJ, McWilliams DJ, et al. An environmental scan for early mobilization practices in US ICUs. Crit Care Med 2015;43:2360-69.

11. Castro E, Turcinovic M, Platz J, Law I. Early mobilization: changing the mindset. Crit Care Nurse 2015;35:e1-6. 
12. Morris PE, Goad A, Thompson C, et al. Early intensive care unit mobility therapy in the treatment of acute respiratory failure. Crit Care Med 2008;36:2238-43.

13. Patel BK, Pohlman AS, Hall JB, Kress JP. Impact of early mobilization on glycemic control and ICU-acquired weakness in critically ill patients who are mechanically ventilated. Chest 2014;146:583-89.

14. Eakin MN, Ugbah L, Arnautovic T, et al. Implementing and sustaining an early rehabilitation program in a medical intensive care unit: a qualitative analysis. J Crit Care 2015;30:698-704.

15. Bauman KA, Hyzy RC. ICU 2020: Five interventions to revolutionize quality of care in the ICU. J Intensive Care Med 2014;29:13-21.

16. Lord RK, Mayhew CR, Korupolu R, et al. ICU early physical rehabilitation programs: financial modeling of cost savings. Crit Care Med 2013;4:717-24.

17. Grammatopoulou E, Charmpas TN, Strati EG, et al. The scope of physiotherapy services provided in public ICUs in Greece: A pilot study. Physiother Theory Pract 2017;33:138-46.

18. Bailey PP, Miller III RR, Clemmer TP. Culture of early mobility in mechanically ventilated patients. Crit Care Med 2009;37:S429-35.

19. Dubb R, Nydahl P, Hermes C, et al. Barriers and strategies for early mobilization of patients in intensive care units. Ann Am Thorac Soc 2016;13:724-30.

20. Zhang L, Hu W, Cai Z, et al. Early mobilization of critically ill patients in the intensive care unit: A systematic review and meta-analysis. PloS One 2019;14:e0223185.

21. Eysenbach, G. Improving the quality of web surveys: the Checklist for Reporting Results of Internet E-Surveys (CHERRIES). J Med Internet Res 2004;6:e34.

22. Bakhru RN, McWilliams DJ, Wiebe DJ, et al. Intensive care unit structure variation and implications for early mobilization practices. An international survey. An Am Thorac Soc 2014;13:1527-37.

23. Li J, Zhan QY, Liang ZA, et al. Respiratory care practices and requirements for respiratory therapists in Beijing intensive care units. Respir Care 2012;57:370-6.

24. Malone D, Ridgeway K, Nordon-Craft A, et al. Physical therapist practice in the intensive care unit: results of a national survey. Phys Ther 2015;95:1335-44.

25. TEAM Study Investigators. Early mobilization and recovery in mechanically ventilated patients in the ICU: a bi-national, multi-centre, prospective cohort study. Crit Care 2015;19:81.

26. Nydahl P, Ruhl AP, Bartoszek G, et al. Early mobilization of mechanically ventilated patients: a 1-day point-prevalence study in Germany. Crit Care Med 2014;42:1178-86.

27. Anekwe DE, Koo KKY, de Marchie M, et al. Interprofessional survey of perceived barriers and facilitators to early mobilization of critically ill patients in Montreal, Canada. J Int Care Med 2019;34:218-26.

28. Koo KK, Choong K, Cook DJ, et al. Early mobilization of critically ill adults: a survey of knowledge, perceptions and practices of Canadian physicians and physiotherapists. CMAJ Open 2016;4:E448.

29. Holdsworth C, Haines KJ, Francis JJ, et al. Mobilization of ventilated patients in the intensive care unit: an elicitation study using the theory of planned behavior. J Crit Care 2015;30:1243-50.

30. Leditschke IA, Green M, Irvine J, et al. What are the barriers to mobilizing intensive care patients? Cardiopulm Phys Ther J 2012;23:26.

31. Connolly BA, Bronwen A, Douiri A, et al. Low levels of physical activity during critical illness and weaning: The evidencereality gap. J Int Care Med 2019;34:818-27.

32. Park YH, Ko RE, Kang D, et al. Relationship between use of rehabilitation resources and ICU readmission and ER visits in ICU survivors: the Korean ICU National Data Study 20082015. J Korean Med Sci 2020;35:e101.

33. Wang TH, Wu CP, Wang LY. Chest physiotherapy with early mobilization may improve extubation outcome in critically ill patients in the intensive care units. Clin Respir J 2018;12:2613-21.

34. Hodgson C, Needham D, Haines K, et al. Feasibility and interrater reliability of the ICU Mobility Scale. Heart Lung 2014;43:19-24

35. Harris CL, Shahid S. Physical therapy-driven quality improvement to promote early mobility in the intensive care unit. Proc (Bayl Univ Med Cent) 2014;27:203-7

36. Damluji A, Zanni JM, Mantheiy E, et al. Safety and feasibility of femoral catheters during physical rehabilitation in the intensive care unit. J Crit Care 2013;28:535.e9-15.

37. Toonstra AL, Zanni JM, Sperati CJ, et al. Feasibility and safety of physical therapy during continuous renal replacement therapy in the intensive care unit. Ann Am Thorac Soc 2016;13:699-704.

38. Abrams D, Javidfar J, Farrand E, et al. Early mobilization of patients receiving extracorporeal membrane oxygenation: a retrospective cohort study. Crit Care 2014;18:R38.

39. Herridge MS, Tansey CM, Matté A, et al. Functional disability 5 years after acute respiratory distress syndrome. N Eng J Med 2011;364:1293-304.

40. Dinglas VD, Colantuoni E, Ciesla N, et al. Occupational therapy for patients with acute lung injury: factors associated with time to first intervention in the intensive care unit. Am J Occup Ther 2013;67:355-62.

41. De Jonghe B, Bastuji-Garin S, Fangio P, et al. Sedation algorithm in critically ill patients without acute brain injury. Crit Care Med 2005;33:120-27.

42. Kress JP, Pohlman AS, O'Connor MF, Hall JB. Daily interruption of sedative infusions in critically ill patients undergoing mechanical ventilation. N Eng J Med 2000;342:1471-7.

43. Inouye SK, Bogardus Jr ST, Charpentier PA, et al. A multicomponent intervention to prevent delirium in hospitalized older patients. N Eng J Med 1999;340:669-76.

44. Balas MC, Vasilevskis EE, Olsen KM, et al. Effectiveness and safety of the awakening and breathing coordination, delirium monitoring/management, and early exercise/mobility (ABCDE) bundle. Crit Care Med 2014;42:1024-36.

45. Bronstein LR. A model for interdisciplinary collaboration. Social work 2003;48:297-306.

46. Jolley SE, Dale CR, Hough CL. Hospital-level factors associated with report of physical activity in patients on mechanical ventilation across Washington State. Ann Am Thorac Soc 2015;12:209-15.

47. Pronovost P, Berenholtz S, Needham D. Translating evidence into practice: a model for large scale knowledge translation. $\mathrm{Br}$ Med J 2008;337:963-65.

48. Needham DM, Korupolu R, Zanni JM, et al. Early physical medicine and rehabilitation for patients with acute respiratory failure: a quality improvement project. Arch Phys Med Rehabil 2010;91:536-42.

49. Engel HJ, Tatebe S, Alonzo PB, et al. Physical therapist-established intensive care unit early mobilization program: quality improvement project for critical care at the University of California San Francisco Medical Center. Phys Ther 2013;93:975-85.

50. Pronovost PJ, Needham DM, Waters $\mathrm{H}$, et al. Intensive care unit physician staffing: financial modeling of the Leapfrog standard. Crit Care 2004;32:1247-53. 